\title{
Szegö limit Theorem on the Lattice
}

\author{
Jitendriya Swain \\ Department of Mathematics \\ IIT, Guwahati \\ e-mail : jitumath@iitg.ernet.in \\ and \\ M Krishna \\ The Institute of Mathematical Sciences \\ Taramani, Chennai 600113 \\ e-mail: krishna@imsc.res.in
}

August 6, 2021

\begin{abstract}
In this paper, we prove a Szegö type limit theorem on $\ell^{2}\left(\mathbb{Z}^{d}\right)$. We consider operators of the form $H=\Delta+V, V$ multiplication by a positive sequence $\left\{V(n), n \in \mathbb{Z}^{d}\right\}$ with $V(n) \rightarrow \infty,|n| \rightarrow \infty$ on $\ell^{2}\left(\mathbb{Z}^{d}\right)$ and $\pi_{\lambda}$ the orthogonal projection of $\ell^{2}\left(\mathbb{Z}^{d}\right)$ on to the space of eigenfunctions of $H$ with eigenvalues $\leq \lambda$. We take $B$ to be a pseudo difference operator of order zero with symbol $b(x, n),(x, n) \in \mathbb{T}^{d} \times \mathbb{Z}^{d}$ and show that for nice functions $f$

$$
\lim _{\lambda \rightarrow \infty} \operatorname{Tr}\left(f\left(\pi_{\lambda} B \pi_{\lambda}\right)\right) / \operatorname{Tr}\left(\pi_{\lambda}\right)=\lim _{\lambda \rightarrow \infty} \frac{1}{(2 \pi)^{d}} \frac{\sum_{V(n) \leq \lambda} \int_{\mathbb{T}^{d}} f(b(x, n)) d x}{\sum_{V(n) \leq \lambda} 1} .
$$
\end{abstract}

\section{Introduction}

In this paper we show a Szegö type theorem on the lattice and give some application to random operators.

The classical theorem of Szegö is stated as follows: Let $P_{n}$ be the orthogonal projection of $L^{2}[0,2 \pi]$ onto the linear subspace spanned by the 
functions $\left\{e^{i m \theta}: 0 \leq m \leq n ; 0 \leq \theta<2 \pi\right\}$. For a positive function $f \in \mathcal{C}^{1+\alpha}[0,2 \pi], \alpha>0$ the operator $T_{f}$ defined by the operator of multiplication by the function $f$ on $L^{2}[0,2 \pi]$ the following result holds

$$
\lim _{n \rightarrow \infty} \frac{1}{n+1} \log \operatorname{det} P_{n} T_{f} P_{n}=\frac{1}{2 \pi} \int_{0}^{2 \pi} \log f(\theta) d \theta .
$$

The above result is well known as Szegö limit theorem. We refer to [8, 4] for details and related results. In fact, Szegö limit theorem is a special case of a more general result proved by Szegö (see [4]) in section 5.3 as follows. Let $f$ be a bounded, real valued integrable function and $\left\{\lambda_{i}^{n}\right\}_{i=1}^{n}$ be the eigenvalues of $P_{n} T_{f} P_{n}$. Then for any continuous function $F$ on [inf $\left.f, \sup f\right]$ it was proved in (see [4, sect. 5.3) that

$$
\lim _{n \rightarrow \infty} \frac{1}{n} \sum_{i=1}^{n} F\left(\lambda_{i}^{n}\right)=\frac{1}{2 \pi} \int_{0}^{2 \pi} F(f(\theta)) d \theta .
$$

Notice that the left hand side here can be seen to be the limit of

$$
\operatorname{Tr}\left(F\left(P_{n} T_{f} P_{n}\right)\right) / \operatorname{Tr}\left(P_{n}\right)
$$

and that $e^{i m \theta}$ is an eigenfunction of $\Delta=-\frac{d^{2}}{d x^{2}}$, so, one can view the above results on $L^{2}[0,2 \pi]$ as a special cases of Szegö limit theorem for the LaplaceBeltrami operator or more generally one can consider such results for pseudo differential operators on compact manifolds.

In [11], Zelditch considered a Schrödinger operator on $\mathbb{R}^{n}$ of the form $H=-\frac{1}{2} \Delta+V$, where $V$ is a smooth positive function which grows like $V_{0}|x|^{k}, k>0$. To establish a Szegö type theorem, as we can see from the above, we need to consider ratios of distribution functions associated to different measures and compare their behaviour asymptotically.

Such limits are computed using Tauberian theorems where some transforms of these measures are considered and limits taken for such transforms. While Zelditch [11] used the Laplace transform (via Karamata's Tauberian theorem ([10],p-192), Robert [6] suggested the use of Stieltjes transform (via Keldysh Tauberian theorem[1]). The application of Keldysh theorem requires one of the measures $\mu$ or $\nu$ to be absolutely continuous. We don't have this feature in our problem, stated below, so we use the Tauberian theorem of Grishin-Poedintseva theorem 3.4 (see [5]) in combination with a theorem of Laptev-Safarov theorem 3.5 (see [2]) that obtains some error estimates to prove our main theorem (Theorem 1.1). 
There is an extensive work on the Szegö's theorem associated with orthogonal polynomials in $L^{2}(\mathbb{T}, d \mu)$ with $\mu$ some probability measure on $\mathbb{T}$, we refer to the monumental work of Barry Simon [9] for the details.

We however concentrate on higher dimensions where not much is known and to our knowledge our results are new in the lattice case.

We consider operators of the form

$$
H=\Delta+V
$$

on $\ell^{2}\left(\mathbb{Z}^{d}\right)$, where $\Delta$ is the positive operator $(\Delta u)(n)=\sum_{|n-j|=1} u(j)+2 d u(n)$. We take $V$ is multiplication by a positive sequence

$$
V(n)=\left\{\begin{array}{l}
1 \\
|n|^{k} \quad, \quad k>0
\end{array}\right.
$$

we chose the value of $V(n)$ to be 1 at the origin to make $V$ strictly positive.

Then $H$ is positive and has discrete spectrum, which is seen by noting that $(H-i)^{-1}$ is compact in view of the choice of $V$. We denote the spectral projection of $H$ by $E_{H}()$ and set $\pi_{\lambda}=E_{H}((0, \lambda])$. Then clearly $\pi_{\lambda}$ is finite rank for each $\lambda$.

For a bounded self-adjoint operator $B$ we set $K=\bigcup_{0 \leq t \leq 1} \sigma(B) \subset \mathbb{R}$, $L^{2}\left(\mathbb{T}^{d}\right)=L^{2}\left(\mathbb{T}^{d}, \frac{d x}{(2 \pi)^{d}}\right)$. Then our main theorems are the following.

Theorem 1.1. Let $H$ and $V$ be as in equation (1, 2). Let $b$ be a bounded real valued measurable function on $\mathbb{T}^{d}$, let $M_{b}$ be the operator of multiplication by $b$ on $L^{2}\left(\mathbb{T}^{d}\right)$ and $B$ its unitary equivalent on $\ell^{2}\left(\mathbb{Z}^{d}\right)$ under the Fourier Series. Assume that there is a $0<\kappa<1$ such that $H^{-\kappa}[H, B]$ is bounded. Then for all $f \in C(K)$, we have

$$
\lim _{\lambda \rightarrow \infty} \frac{\operatorname{Tr}\left(f\left(\pi_{\lambda} B \pi_{\lambda}\right)\right)}{\operatorname{Tr}\left(\pi_{\lambda}\right)}=\frac{1}{(2 \pi)^{d}} \int f(b(x)) d x .
$$

We recollect some facts on toroidal symbols from Rhuzanski-Turunen [7] below. A linear operator $A$ on $L^{2}\left(\mathbb{T}^{d}\right)$ associated with symbols $\sigma(x, n),(x, n) \in$ $\mathbb{T}^{d} \times \mathbb{Z}^{d}$, (the reader should note that the lattice variable $\xi$ appearing in [7] should be replaced by $m, n$ etc in our notation) is defined by

$$
(A \phi)(x)=\sum_{n \in \mathbb{Z}^{d}} \frac{1}{(2 \pi)^{d}} \int e^{i(x-y) n} \sigma(x, n) \phi(x) d x
$$


where $\phi \in C^{\infty}\left(\mathbb{T}^{d}\right)$ and the symbol $\sigma \in C^{\infty}\left(\mathbb{T}^{d} \times \mathbb{Z}^{d}\right),\left(a \in C^{\infty}\left(\mathbb{T}^{d} \times \mathbb{Z}^{d}\right)\right.$ means $a(\cdot, n) \in C^{\infty}\left(\mathbb{T}^{d}\right)$ for all $\left.n \in \mathbb{Z}^{d}\right)$. Then $A$ extends to a bounded linear operator and via the unitary isomorphism implemented by the Fourier series (call it $U^{*}$ ) between $L^{2}\left(\mathbb{T}^{d}\right)$ and $\ell^{2}\left(\mathbb{Z}^{d}\right)$, gives also a bounded operator on $\ell^{2}\left(\mathbb{Z}^{d}\right)$. Thus associated to every symbol $\sigma \in C^{\infty}\left(\mathbb{T}^{d} \times \mathbb{Z}^{d}\right)$, there is a bounded operator on $\ell^{2}\left(\mathbb{Z}^{d}\right)$ and such an operator is self-adjoint whenever $\sigma$ is real valued. We will say that $b(x, n)$ is the symbol of a bounded linear operator $B$ on $\ell^{2}\left(\mathbb{Z}^{d}\right)$ to mean that the equation (44) is valid by setting $A=$ $U B U^{*}, \sigma(x, n)=b(x, n)$ and $B$ is an appropriate bounded linear extension in $\ell^{2}\left(\mathbb{Z}^{d}\right)$. The difference operator $\Delta_{n_{j}}$ is given by $\left(\Delta_{n_{j}} \phi\right)(m)=\phi(m+$ $\left.e_{j}\right)-\phi(m), e_{j}$ being the unit vector in the $j^{\text {th }}$ direction in $\mathbb{Z}^{d}$ and acts on symbols in the second variable. Denoting $\mathbb{N}_{0}=\mathbb{N} \cup\{0\}$, and a multi index $\alpha=\left(\alpha_{1}, \ldots, \alpha_{d}\right) \geq 0$ to mean $\alpha_{j} \in \mathbb{N}_{0}, j=1, \ldots, d$. Then the difference operator $\Delta_{n}^{\alpha}=\Delta_{n_{1}}^{\alpha_{1}} \Delta_{n_{2}}^{\alpha_{2}} \cdots \Delta_{n_{d}}^{\alpha_{d}}$. Let $\langle n\rangle=\left(1+|n|^{2}\right)^{\frac{1}{2}}$. The class of rapidly decreasing sequences is given by

$$
\mathcal{S}\left(\mathbb{Z}^{d}\right)=\left\{\phi(n): \forall M \in \mathbb{N},|\phi(n)| \leq C_{\phi, M}\langle n\rangle^{-M}\right\} .
$$

Given these definitions, for any $m \in \mathbb{R}, 0 \leq \rho, \delta \leq 1$, the toroidal symbol class $S_{\rho, \delta}^{m}\left(\mathbb{T}^{d} \times \mathbb{Z}^{d}\right)$ is defined as all $\sigma \in C^{\infty}\left(\mathbb{T}^{d} \times \mathbb{Z}^{d}\right)$ such that

$$
\left|\Delta_{n}^{\alpha} D_{x}^{\beta} \sigma(x, n)\right| \leq C_{\sigma \alpha \beta m}\langle n\rangle^{m-\rho|\alpha|+\delta|\beta|}, \forall(x, n) \in \mathbb{T}^{d} \times \mathbb{Z}^{d} \text { and } \alpha, \beta \in \mathbb{N}_{0}^{d} .
$$

The class $S_{1,0}^{0}$ is denoted simply by $S_{1,0}$. Let us define a subclass of symbols where all the derivatives in $x$ also have uniform bounds.

$$
S_{1,0, \infty}^{m}\left(\mathbb{T}^{d} \times \mathbb{Z}^{d}\right)=\left\{\sigma \in S_{1,0}^{m}\left(\mathbb{T}^{d} \times \mathbb{Z}^{d}\right): C_{\sigma \alpha \beta m} \text { are independent of } \beta\right\} .
$$

So we denote $S_{1,0, \infty}^{0}$ by $S_{1,0, \infty}$.

Given this framework, we have our next theorem.

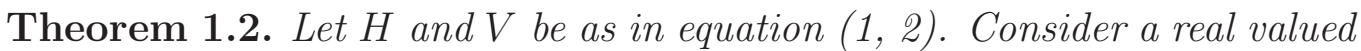
$b \in S_{1,0, \infty}\left(\mathbb{T}^{d} \times \mathbb{Z}^{d}\right)$ and let $B$ be the associated bounded self-adjoint operator on $\ell^{2}\left(\mathbb{Z}^{d}\right)$. Assume that there is a $0<\kappa<1$ such that $H^{-\kappa}[H, B]$ is bounded. Then for all $f \in C(K)$, we have

$$
\lim _{\lambda \rightarrow \infty} \frac{\operatorname{Tr}\left(f\left(\pi_{\lambda} B \pi_{\lambda}\right)\right)}{\operatorname{Tr}\left(\pi_{\lambda}\right)}=\lim _{\lambda \rightarrow \infty} \frac{\frac{1}{(2 \pi)^{d}} \int \sum_{V(n) \leq \lambda} f(b(x, n)) d x}{\sum_{V(n) \leq \lambda} 1} .
$$

The function $\cos \left(x+\gamma_{n}\right)$ with $\gamma_{n} \rightarrow 0$ as $|n| \rightarrow \infty$ is in $S_{1,0, \infty}\left(\mathbb{T}^{d} \times \mathbb{Z}^{d}\right.$ for example, so the class is non-empty. 


\section{The Proofs:}

We denoted by $\# S$ the cardinality of the set $S$ in the following. Consider $H, V$ as in equations (1), 2).

Then for $\lambda>0$ the operator $H+\lambda$ is also positive. $H$ has discrete spectrum and so the bounded operator $(H+\lambda)^{-1}$ is compact. By taking proper power $k$ we can make it trace class, so let us for the sake of simplicity assume $k>d$ so this operator is trace class.

Given this if we consider the operator $V$ and $(V+\lambda)$, then these two are operators of multiplication on $\ell^{2}\left(\mathbb{Z}^{d}\right)$, they both have discrete spectrum and are positive so we also have, the our choice of $k$, that $(V+\lambda)^{-m}$ and $(H+\lambda)^{-m}$ are also trace class for some $m \in \mathbb{N}$.

Lemma 2.1. Consider $V$ and $H$ self-adjoint operators as given in equations (2):1). Then for $m \in \mathbb{N}$ for which $(V+\lambda)^{-m}$ is trace class, $(H+\lambda)^{-m}$ is also trace class and we have,

$$
\left|\frac{\operatorname{Tr}\left((H+\lambda)^{-m}\right)}{\operatorname{Tr}\left((V+\lambda)^{-m}\right)}-1\right| \rightarrow 0
$$

as $\lambda \rightarrow \infty$.

Proof: Since $\Delta$ is bounded and $(V+\lambda)^{-1}$ is bounded and positive we can write

$$
(H+\lambda)=(V+\lambda)^{\frac{1}{2}}\left((V+\lambda)^{-\frac{1}{2}} \Delta(V+\lambda)^{-\frac{1}{2}}+1\right)(V+\lambda)^{\frac{1}{2}} .
$$

Since $K_{\lambda}=(V+\lambda)^{-\frac{1}{2}} \Delta(V+\lambda)^{-\frac{1}{2}}$ is bounded and has norm smaller than 1 for large $\lambda$, we have expansion

$$
\left.(H+\lambda)^{-m}=(V+\lambda)^{-m}+(V+\lambda)^{-\frac{m}{2}}\left(\left(1+K_{\lambda}\right)^{-m}-1\right)\right)(V+\lambda)^{-\frac{m}{2}} .
$$

This equality shows that $(H+\lambda)^{-m}$ is trace class whenever $(V+\lambda)^{-m}$ is trace class, so we take trace on both sides of the above equation. We then use the property of trace, the inequality $|\operatorname{Tr}(B C B)| \leq\|C\| \operatorname{Tr}\left(B^{2}\right)$, when $B$ is positive trace class and $C$ is bounded and that $\left(1+K_{\lambda}\right)^{-1}$ is bounded by 1 , by the positivity of $K_{\lambda}$, to get

$$
\begin{aligned}
& \left|\operatorname{Tr}\left((H+\lambda)^{-m}\right)-\operatorname{Tr}\left((V+\lambda)^{-m}\right)\right| \\
& \left.\quad=\operatorname{Tr}\left((V+\lambda)^{-\frac{m}{2}}\left(\left(1+K_{\lambda}\right)^{-m}-1\right)\right)(V+\lambda)^{-\frac{m}{2}}\right) \mid \\
& \left.\leq \operatorname{Tr}\left((V+\lambda)^{-m}\right) \|\left(\left(1+K_{\lambda}\right)^{-m}-1\right)\right)\|\leq m\| K_{\lambda} \| \operatorname{Tr}\left((V+\lambda)^{-m}\right) \\
& \leq \operatorname{Tr}\left((V+\lambda)^{-m}\right) m\|\Delta\|\left\|(V+\lambda)^{-1}\right\|^{m}
\end{aligned}
$$


Therefore,

$$
\left|\frac{\operatorname{Tr}\left((H+\lambda)^{-m}\right)}{\operatorname{Tr}\left((V+\lambda)^{-m}\right)}-1\right| \leq 4 d m\left\|(V+\lambda)^{-1}\right\|^{m}
$$

which gives the lemma as $\lambda$ goes to $\infty$.

Let $E_{A}$ denotes the projection value spectral measure of $A$. Denote the distribution functions of the measures $\operatorname{Tr}\left(E_{H}(\cdot)\right)$ and $\operatorname{Tr}\left(E_{V}(\cdot)\right)$ respectively by $\phi_{H}$ and $\phi_{V}$. Then we have

$$
\phi_{H}(\lambda)=\operatorname{Tr}\left(\pi_{\lambda}\right), \quad \phi_{V}(\lambda)=\#\{n: V(n) \in(0, \lambda]\} .
$$

Then Lemma 2.1 immediately gives us the Weyl formula for the functions $\operatorname{Tr}\left(\pi_{\lambda}\right)$ as a corollary, where we denote by $[r]$ the largest integer smaller than or equal to $r$.

Corollary 2.2. Consider $V$ and $H$ self-adjoint operators as given in equations (1]:2). We have the following asymptotics:

1. $\phi_{V}$ is multiplicatively continuous.

2.

$$
\operatorname{Tr}\left(\pi_{\lambda}\right)-\#\{n: V(n) \in(0, \lambda]\} \approx o(\#\{n: V(n) \in(0, \lambda]\}), \text { as } \lambda \rightarrow \infty .
$$

3.

$$
\operatorname{Tr}\left(\pi_{\lambda}\right)=2^{d}[\lambda]^{\frac{d}{k}}+o\left(\lambda^{\frac{d}{k}}\right), \text { as } \lambda \rightarrow \infty .
$$

4.

$$
\sup _{\mu \leq \lambda}\left(\operatorname{Tr}\left(\pi_{\mu+r}\right)-\operatorname{Tr}\left(\pi_{\mu}\right)\right) \leq \operatorname{Tr}\left(\pi_{\lambda}\right)\left(\frac{d}{k} \frac{[r]+1}{[\lambda]}+O\left(\frac{1}{[\lambda]}\right)\right), \text { as } \lambda \rightarrow \infty .
$$

Proof: (1) The function $\phi_{V}$ is given by

$$
\begin{aligned}
\phi_{V}(\lambda) & =\#\{n: V(n) \leq \lambda\}=\#\left\{n:|n|^{k} \leq \lambda\right\} \\
& =\#\left\{n:|n| \leq[\lambda]^{1 / k}\right\}=\left(2[\lambda]^{\frac{1}{k}}+1\right)^{d} .
\end{aligned}
$$

Therefore clearly $\lim _{\lambda \rightarrow \infty} \lim _{\tau \rightarrow 1} \phi_{V}(\tau \lambda) / \phi_{V}(\lambda)=1$. On the other hand, using the notation $(r)$ for the fractional part of $r$, we see from equation (8) 
that

$$
\begin{aligned}
\frac{\phi_{V}(\tau \lambda)}{\phi_{V}(\lambda)} & =\frac{\left(2[\tau \lambda]^{\frac{1}{k}}+1\right)^{d}}{\left(2[\lambda]^{\frac{1}{k}}+1\right)^{d}}=\frac{\left(2(\tau \lambda-(\tau \lambda))^{\frac{1}{k}}+1\right)^{d}}{\left(2(\lambda-(\lambda))^{\frac{1}{k}}+1\right)^{d}} \\
& =\tau^{d / k} \frac{\left(2\left(1-\frac{(\tau \lambda)}{\tau \lambda}\right)^{1 / k}+\frac{1}{|\tau \lambda|^{1 / k}}\right)^{d}}{\left(2\left(1-\frac{(\lambda)}{\lambda}\right)^{1 / k}+\frac{1}{\lambda^{1 / k}}\right)^{d}}
\end{aligned}
$$

Taking the limit over $\lambda$ first and then over $\tau$ we see that

$$
\lim _{\tau \rightarrow 1} \lim _{\lambda \rightarrow \infty} \frac{\phi_{V}(\tau \lambda)}{\phi_{V}(\lambda)}=1 .
$$

Lemma 2.1 implies that

$$
\int_{0}^{\infty} \frac{\lambda^{m}}{(\lambda+u)^{m}} d \phi_{H}(u) / \int_{0}^{\infty} \frac{\lambda^{m}}{(\lambda+u)^{m}} d \phi_{V}(u) \rightarrow 1 \text {, as } \lambda \rightarrow \infty .
$$

(2) The distribution function This in turn implies, by Theorem 3.4 of Grishin-Poedintseva, that

$$
\phi_{H}(\lambda) / \phi_{V}(\lambda) \rightarrow 1 \text {, as } \lambda \rightarrow \infty \text {. }
$$

Then (2) follows.

(3) This follows from (2) and equation (8) .

(4) Using the asymptotics (3), bounding the terms in the ratio $\frac{\operatorname{Tr}\left(\pi_{\mu+r}\right)}{\operatorname{Tr}\left(\pi_{\lambda}\right)}$ and keeping the possibility that $r$ also goes to infinity we get this estimate.

This corollary implies that $\phi_{H}$ is also a multiplicatively continuous function from the following Lemma.

Lemma 2.3. The function $\phi_{H}$ considered above is multiplicatively continuous at infinity.

Proof: We will show that if $\varphi, \chi$ are two distribution functions satisfying

$$
\lim _{r \rightarrow \infty} \frac{\varphi(r)}{\chi(r)}=1
$$

then $\varphi$ is multiplicatively continuous whenever $\chi$ is. Clearly

$$
\lim _{r \rightarrow \infty} \lim _{\tau \rightarrow 1} \frac{\varphi(\tau r)}{\varphi(r)}=\lim _{r \rightarrow \infty} \frac{\varphi(r)}{\varphi(r)}=1 .
$$


Now consider

$$
\begin{aligned}
\lim _{\tau \rightarrow 1} \lim _{r \rightarrow \infty} \frac{\varphi(\tau r))}{\varphi(r)} & =\lim _{\tau \rightarrow 1} \lim _{r \rightarrow \infty} \frac{\frac{\varphi(\tau r))}{\chi(\tau r)}}{\frac{\varphi(r)}{\chi(r)}} \\
& =\lim _{\tau \rightarrow 1} 1=1,
\end{aligned}
$$

where in the last step we used the assumption on $\phi / \chi$ and the fact that $\chi$ is multiplicatively continuous. Since $\phi_{V}$ is multiplicatively continuous, the above result together with equation (10) now shows that $\phi_{H}$ is multiplicatively continuous.

Lemma 2.4. Suppose $B$ is a bounded positive operator on $\ell^{2}\left(\mathbb{Z}^{d}\right)$, then, for $m \in \mathbb{N}$ for which $(V+\lambda)^{-m}$ (and hence $(H+\lambda)^{-m}$ ) is trace class, we have,

$$
\left|\frac{\operatorname{Tr}\left(B(H+\lambda)^{-m}\right)}{\operatorname{Tr}\left(B(V+\lambda)^{-m}\right)}-1\right| \rightarrow 0
$$

as $\lambda \rightarrow \infty$.

Proof: The proof is similar to that in the above lemma, except that we have to do a bit more of algebra in handling the error term, namely, using equation (6) we write

$$
\begin{aligned}
\operatorname{Tr}\left(B(H+\lambda)^{-m}\right) & =\operatorname{Tr}\left(B(V+\lambda)^{-m}\right) \\
& \left.+\operatorname{Tr}\left(B(V+\lambda)^{-\frac{m}{2}}\left(\left(1+K_{\lambda}\right)^{-m}-1\right)\right)(V+\lambda)^{-\frac{m}{2}}\right) .
\end{aligned}
$$

we set $W_{\lambda}=(V+\lambda)^{-\frac{m}{2}} B(V+\lambda)^{-\frac{m}{2}}$ which is a positive trace class, so we rewrite the error term as

$$
\begin{aligned}
& \left.\mid \operatorname{Tr}\left(B(V+\lambda)^{-\frac{m}{2}}\left(\left(1+K_{\lambda}\right)^{-m}-1\right)\right)(V+\lambda)^{-\frac{m}{2}}\right) \mid \\
& \left.\leq \mid \operatorname{Tr}\left((V+\lambda)^{-\frac{m}{2}} B(V+\lambda)^{-\frac{m}{2}}\left(\left(1+K_{\lambda}\right)^{-m}-1\right)\right)\right) \mid \\
& \left.\mid \operatorname{Tr}\left(W_{\lambda}^{\frac{1}{2}}\left(\left(1+K_{\lambda}\right)^{-m}-1\right) W_{\lambda}^{\frac{1}{2}}\right)\right) \mid \\
& \leq \operatorname{Tr}\left(W_{\lambda}\right)\left\|\left(\left(1+K_{\lambda}\right)^{-m}-1\right)\right\| \leq \operatorname{Tr}\left(B(V+\lambda)^{-m}\right) m\|\Delta\|\left\|(V+\lambda)^{-1}\right\|^{m} .
\end{aligned}
$$

The rest of the proof is as in the Lemma 2.1 using the above estimate.

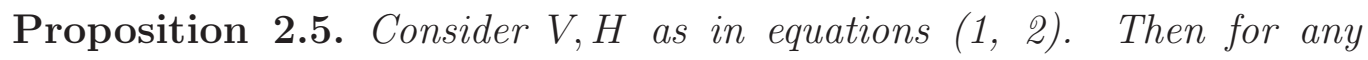
bounded positive operator $B$ and $m \in \mathbb{N}$ be such that $(V+\lambda)^{-m}$ is trace class. Then we have 
(i) The following equality is valid in the sense that if one of the limits exists then the other also does and the limits are the same.

$$
\lim _{\lambda \rightarrow \infty} \frac{\operatorname{Tr}\left(B(H+\lambda)^{-m}\right)}{\operatorname{Tr}\left((H+\lambda)^{-m}\right)}=\lim _{\lambda \rightarrow \infty} \frac{\operatorname{Tr}\left(B(V+\lambda)^{-m}\right)}{\operatorname{Tr}\left((V+\lambda)^{-m}\right)} .
$$

(ii) If in addition $B$ comes from an operator of multiplication by a function $b$ on $L^{2}\left(\mathbb{T}^{d}\right)$, then the limits in (i) exist.

Proof: (i) For each $\lambda$ we have the equality

$$
\frac{\left(\frac{\operatorname{Tr}\left(B(H+\lambda)^{-m}\right)}{\operatorname{Tr}\left(B(V+\lambda)^{-m}\right)}\right)}{\left(\frac{\operatorname{Tr}\left((H+\lambda)^{-m}\right)}{\operatorname{Tr}\left((V+\lambda)^{-m}\right)}\right)}=\frac{\left(\frac{\operatorname{Tr}\left(B(H+\lambda)^{-m}\right)}{\operatorname{Tr}\left((H+\lambda)^{-m}\right)}\right)}{\left(\frac{\operatorname{Tr}\left(B(V+\lambda)^{-m}\right)}{\operatorname{Tr}\left((V+\lambda)^{-m}\right)}\right)} .
$$

Lemma 2.1 and Lemma 2.4 imply that the left hand side has limit 1, hence the right hand side limit exists and equals 1 . Therefore if either the numerator or the denominator in the fraction in the right hand side has a limit, then the other also has a limit and they both agree which implies the proposition.

(ii) In the case when $B$ comes from an operator of multiplication by a function $b$ on $L^{2}\left(\mathbb{T}^{d}\right)$ we have, using the Fourier series to compute $\left\langle\delta_{n}, B \delta_{n}\right\rangle$ for any $n$,

$$
\begin{aligned}
\operatorname{Tr}\left(B(V+\lambda)^{-m}\right) & =\sum_{n \in \mathbb{Z}^{d}}\left\langle\delta_{n}, B \delta_{n}\right\rangle(V(n)+\lambda)^{-m} \\
& =\left(\frac{1}{(2 \pi)^{d}} \int b(x) d x\right) \sum_{n \in \mathbb{Z}^{d}}(V(n)+\lambda)^{m} \\
& =\left(\frac{1}{(2 \pi)^{d}} \int b(x) d x\right) \operatorname{Tr}\left((V+\lambda)^{-m}\right) .
\end{aligned}
$$

Therefore we have for each $\lambda$,

$$
\frac{\operatorname{Tr}\left(B(V+\lambda)^{-m}\right)}{\operatorname{Tr}\left((V+\lambda)^{-m}\right)}=\left(\frac{1}{(2 \pi)^{d}} \int b(x) d x\right),
$$

so the limit of the left hand side as $\lambda$ goes to infinity exists.

Given these general results, we now use the theorems of Laptev-Safarov (Theorem 3.5) and the Tauberian theorem of Grishin-Poedintseva (Theorem 3.4) to pass onto measures associated with the self adjoint operators $H$ and $V$. 


\section{Proof of Theorem 1.1:}

Since $B$ is bounded self-adjoint $\|B\| \in \sigma(B)$, since $B$ is also positive, from the definition of $K$ it is clear that $[0,\|B\|]=[0,1]\|B\| \subset K$. Since $\left\|\pi_{\lambda} B \pi_{\lambda}\right\| \leq\|B\|$, it is also clear that for each $\lambda, \sigma\left(\pi_{\lambda} B \pi_{\lambda}\right) \subset K$. Also since $K$ is compact by definition, the elements of $C(K)$ can be uniformly approximated by those from $W_{\infty}^{2}(K)$ (the space of all twice continuously differentiable functions equipped with the norm $\left\||f \||=\sum_{j=0}^{2}\left|f^{(j)}\right|_{\infty}\right)$ by StoneWeierstrass, since the latter contains polynomials. Therefore it is enough to prove the theorem for $f \in W_{\infty}^{2}(K)$.

We set $N_{r}(\lambda)=\sup _{\mu \leq \lambda}\left(\operatorname{Tr}\left(\pi_{\mu+r}\right)-\operatorname{Tr}\left(\pi_{\mu}\right)\right), r>0$. Then using the theorem 3.5 we get

$$
\begin{aligned}
& \left|\frac{\operatorname{Tr}\left(f\left(\pi_{\lambda} B \pi_{\lambda}\right)\right)}{\operatorname{Tr}\left(\pi_{\lambda}\right)}-\frac{\operatorname{Tr}\left(\pi_{\lambda} f(B) \pi_{\lambda}\right)}{\operatorname{Tr}\left(\pi_{\lambda}\right)}\right| \\
& \leq \frac{1}{\operatorname{Tr}\left(\pi_{\lambda}\right)} \frac{1}{2}\left\|f^{\prime \prime}\right\| N_{r}(\lambda)\left(\left\|\pi_{\lambda-r} B\right\|^{2}+\frac{\pi^{2} \lambda^{2 \kappa}}{6 r^{2}}\left\|H^{-\kappa} \pi_{\lambda-r}[H, B]\right\|^{2}\right) \\
& \leq C \frac{N_{\lambda^{\kappa}}(\lambda)}{\operatorname{Tr}\left(\pi_{\lambda}\right)} \leq C \frac{\left[\lambda^{\kappa}\right]}{[\lambda]}
\end{aligned}
$$

For getting the last estimate, we take $r=\lambda^{\kappa}$, then by the assumptions on $B$ the term in the parenthesis on the right hand side in the penultimate estimate above is bounded, so using Corollary 2.2(3), we get the last bound. The last term clearly goes to zero as $\lambda \rightarrow \infty$ so it is enough to show

$$
\left|\frac{\operatorname{Tr}\left(\pi_{\lambda} f(B) \pi_{\lambda}\right)}{\operatorname{Tr}\left(\pi_{\lambda}\right)}-\frac{1}{(2 \pi)^{d}} \int f(b(x)) d x\right| \rightarrow 0, \text { as } \lambda \rightarrow \infty
$$

Let $\pi_{V, \lambda}$ denote the spectral projection $E_{V}((0, \lambda])$, then

$$
\operatorname{Tr}\left(\pi_{V, \lambda} f(B) \pi_{V, \lambda}\right)=\sum_{V(n) \leq \lambda}\left\langle\delta_{n}, f(B) \delta_{n}\right\rangle, \text { and } \operatorname{Tr}\left(\pi_{V, \lambda}\right)=\sum_{V(n) \leq \lambda} 1 .
$$

Since, under the Fourier series the basis vectors $\left|\delta_{n}\right\rangle$ go over to the basis $e^{i n \cdot x}$ in $L^{2}\left(\mathbb{T}^{d}\right)$ and $B$ is an operator of multiplication by a bounded positive function $b(x)$ there, we see that

$$
\left\langle\delta_{n}, f(B) \delta_{n}\right\rangle=\frac{1}{(2 \pi)^{d}} \int_{\mathbb{T}^{d}} f(b(x)) d x .
$$


Therefore, for each $\lambda$,

$$
\frac{\operatorname{Tr}\left(\pi_{V, \lambda} f(B) \pi_{V, \lambda}\right)}{\operatorname{Tr}\left(\pi_{V, \lambda}\right)}=\frac{1}{(2 \pi)^{d}} \int_{\mathbb{T}^{d}} f(b(x)) d x .
$$

Therefore it is enough to show that

$$
\lim _{\lambda \rightarrow \infty} \frac{\operatorname{Tr}\left(\pi_{\lambda} f(B) \pi_{\lambda}\right)}{\operatorname{Tr}\left(\pi_{\lambda}\right)}=\lim _{\lambda \rightarrow \infty} \frac{\operatorname{Tr}\left(\pi_{V, \lambda} f(B) \pi_{V, \lambda}\right)}{\operatorname{Tr}\left(\pi_{V, \lambda}\right)}
$$

to prove the theorem. Adding a constant to the function $f$ does not matter in the above, so we can assume without loss of generality that the function $f$ is positive, so $f(B)$ is a positive operator and hence $f(b(x))$ is a positive function on $\mathbb{T}^{d}$.

Now recall the definition of $\phi_{H}, \phi_{V}$ and we set

$$
\begin{aligned}
\phi_{H, f}(\lambda) & =\operatorname{Tr}\left(\pi_{\lambda} f(B) \pi_{\lambda}\right)=\operatorname{Tr}\left(f(B)^{\frac{1}{2}} \pi_{\lambda} f(B)^{\frac{1}{2}}\right), \\
\phi_{V, f}(\lambda) & =\operatorname{Tr}\left(\pi_{V, \lambda} f(B) \pi_{V, \lambda}\right)=\operatorname{Tr}\left(f(B)^{\frac{1}{2}} \pi_{V, \lambda} f(B)^{\frac{1}{2}}\right),
\end{aligned}
$$

In this notation, the claim in the equation is nothing but the convergence

$$
\lim _{\lambda \rightarrow \infty} \frac{\phi_{H, f}(\lambda)}{\phi_{H}(\lambda)}=\lim _{\lambda \rightarrow \infty} \frac{\phi_{V, f}(\lambda)}{\phi_{H}(\lambda)}
$$

This convergence follows if we show, by theorem 3.4 ,

$$
\lim _{r \rightarrow \infty} \frac{\int \frac{\phi_{H, f}(u)}{\left(1+\frac{u}{r}\right)^{m+1}} d u}{\int \frac{\phi_{H}(u)}{\left(1+\frac{u}{r}\right)^{m+1}} d u}=\lim _{r \rightarrow \infty} \frac{\int \frac{\phi_{V, f}(u)}{\left(1+\frac{u}{r}\right)^{m+1}} d u}{\int \frac{\phi_{V}(u)}{\left(1+\frac{u}{r}\right)^{m+1}} d u}
$$

This equality is precisely the content of Proposition 2.5 (where $B$ is replaced by $f(B)$ ), after an integration by parts performed in all the integrals above and using the spectral theorem respectively for $H$ and $V$ to rewrite the integrals as traces.

\section{Proof of Theorem 1.2,}

The proof is as in the proof of theorem 1.1 till equation (17), the difference comes in computing the limit of the right hand side in equation (17). Hence it is enough to compute the limits

$$
\lim _{\lambda \rightarrow \infty} \frac{\operatorname{Tr}\left(\pi_{V, \lambda} f(B) \pi_{V, \lambda}\right)}{\operatorname{Tr}\left(\pi_{V, \lambda}\right)}=\lim _{\lambda \rightarrow \infty} \frac{\sum_{n: V(n) \leq \lambda}\left\langle\delta_{n}, f(B) \delta_{n}\right\rangle}{\sum_{V(n) \leq \lambda} 1} .
$$


when $B$ comes from a symbol $b(x, n)$ and $f$ continuous. The operator $B$ being bounded self-adjoint, continuity of $f$ implies that $f(B)$ is approximated in norm by polynomial functions of $B$, by using the spectral theorem and the Stone-Weierstrass theorem together, which approximation is uniform in $\lambda$ in the above limits, hence by an $\epsilon / 3$ argument, it is enough to consider $f$ to be a polynomial of a fixed degree and further by linearity of the limits it is enough to take $f$ to be a monomial in $B$.

Therefore we need to show that for any $k \in \mathbb{N}$,

$$
\lim _{\lambda \rightarrow \infty} \frac{\sum_{n: V(n) \leq \lambda}\left\langle\delta_{n}, B^{k} \delta_{n}\right\rangle}{\sum_{V(n) \leq \lambda} 1}=\lim _{\lambda \rightarrow \infty} \frac{\sum_{n: V(n) \leq \lambda} \frac{1}{(2 \pi)^{d}} \int(b(x, n))^{k} d x}{\sum_{V(n) \leq \lambda} 1}
$$

when $B$ comes from a symbol $b \in S_{1,0}\left(\mathbb{T}^{d} \times \mathbb{Z}^{d}\right)$ to prove the theorem.

Therefore a simple computation shows that firstly if $\sigma_{B^{k}}(x, n)$ is the symbol associated with $B^{k}$ then,

$$
\left\langle\delta_{n}, B^{k} \delta_{n}\right\rangle=\left\langle U \delta_{n},\left(U B U^{*}\right)^{k} U \delta_{n}\right\rangle=\frac{1}{(2 \pi)^{d}} \int \sigma_{B^{k}}(x, n) d x .
$$

Secondly the symbol $\sigma_{B^{k}}(x, n)$ is given in terms of $b(x, n)$ asymptotically in $n$ using the above relation applied $k$ times on $b$. Using Lemma 2.6 below we see that

$$
\left\langle\delta_{n}, B^{k} \delta_{n}\right\rangle=\frac{1}{(2 \pi)^{d}} \int b(x, n)^{k} d x+\frac{1}{(2 \pi)^{d}} \int E_{k}(x, n) d x
$$

with $\sup _{x}\left|E_{k}(x, n)\right| \rightarrow 0$ as $|n| \rightarrow 0$.

Then computing the limits and using the fact that if a sequence $r_{n}$ goes to zero as $|n|$ goes to infinity then $\left(\sum_{V(n) \leq \lambda} r_{n}\right) / \sum_{V(n) \leq \lambda}$ goes to zero as $\lambda$ goes to infinity, by an $\epsilon / 3$ argument. Therefore using the properties of $E_{k}(x, n)$ stated above together with the Lebesgue dominated convergence theorem, we see that

$$
\begin{aligned}
\lim _{\lambda \rightarrow \infty} \frac{\sum_{n: V(n) \leq \lambda}\left\langle\delta_{n}, B^{k} \delta_{n}\right\rangle}{\sum_{V(n) \leq \lambda} 1} & =\lim _{\lambda \rightarrow \infty} \frac{\sum_{n: V(n) \leq \lambda} \frac{1}{(2 \pi)^{d}} \int(b(x, n))^{k} d x}{\sum_{V(n) \leq \lambda} 1} \\
& +\lim _{\lambda \rightarrow \infty} \frac{\sum_{n: V(n) \leq \lambda} \frac{1}{(2 \pi)^{d}} \int E_{k}(x, n) d x}{\sum_{V(n) \leq \lambda} 1} \\
& =\lim _{\lambda \rightarrow \infty} \frac{\sum_{n: V(n) \leq \lambda} \frac{1}{(2 \pi)^{d}} \int(b(x, n))^{k} d x}{\sum_{V(n) \leq \lambda} 1} .
\end{aligned}
$$


This proves the theorem.

From theorem 4.2 [7], we see that if $A, B$ are linear operators in $L^{2}\left(\mathbb{T}^{d}\right)$ associates with symbols $a, b \in S_{1,0}^{0}\left(\mathbb{T}^{d} \times \mathbb{Z}^{d}\right)$, then $A B$ also comes from a symbol $\sigma(x, n) \in S_{1,0}^{0}\left(\mathbb{T}^{d} \times \mathbb{Z}^{d}\right)$ and

$$
\sigma(x, n) \approx \sum_{\alpha \geq 0} \frac{1}{\alpha !}\left(\Delta_{m}^{\alpha} a(x, n)\right) D_{x}^{(\alpha)} b(x, n)
$$

where $\approx$ means asymptotic in $n$.

Lemma 2.6. Consider a symbol $a(x, n) \in S_{1,0, \infty}\left(\mathbb{T}^{d} \times \mathbb{Z}^{d}\right)$ and let $A$ be the pseudo difference operator on $L^{2}\left(\mathbb{T}^{d}\right)$ associated with it. Then for any $k \in \mathbb{N}$, the symbol $a_{k}(x, n)$ of the operator $A^{k}$ has the asymptotic behaviour

$$
a_{k}(x, n) \approx(a(x, n))^{k}+E_{k}(x, n),
$$

with $E_{k}(x, n) \in S_{1,0, \infty}^{-1}$.

Proof: We will prove this by induction, since for $k=1$ this is trivial with $E_{1}(x, n)=0$. We assume that the Lemma is valid for $a_{k-1}(x, n)$, so we assume that

$$
a_{k-1}(x, n) \approx(a(x, n))^{k-1}+E_{k-1}(x, n), \text { with } E_{k-1} \in S_{1,0, \infty}^{-1} .
$$

We use the composition rule in equation (20) to get

$$
\begin{aligned}
a_{k}(x, n) & \approx \sum_{\alpha \geq 0} \frac{1}{\alpha !}\left(\Delta_{n}^{\alpha} a(x, n)\right) D_{x}^{(\alpha)} a_{k-1}(x, n) \\
& \approx \sum_{\alpha \geq 0} \frac{1}{\alpha !}\left(\Delta_{n}^{\alpha} a(x, n)\right) D_{x}^{(\alpha)}(a(x, n))^{k-1}+\sum_{\alpha \geq 0} \frac{1}{\alpha !}\left(\Delta_{n}^{\alpha} a(x, n)\right) D_{x}^{(\alpha)} E_{k-1}(x, n) \\
& \approx(a(x, n))^{k}+T_{1}(x, n)+T_{2}(x, n),
\end{aligned}
$$

where

$$
\begin{aligned}
& T_{1}(x, n)=\sum_{|\alpha| \geq 1} \frac{1}{\alpha !}\left(\Delta_{n}^{\alpha} a(x, n)\right) D_{x}^{(\alpha)}(a(x, n))^{k-1}, \\
& T_{2}(x, n)=\sum_{\alpha \geq 0} \frac{1}{\alpha !}\left(\Delta_{n}^{\alpha} a(x, n)\right) D_{x}^{(\alpha)} E_{k-1}(x, n) .
\end{aligned}
$$


We set $E_{k}(x, n)=T_{1}(x, n)+T_{2}(x, n)$ and prove its properties stated in the assumption. We recall the relation

$$
\Delta_{n}^{\alpha} \sigma(n)=\sum_{\beta \leq \alpha}(-1)^{|\alpha-\beta|}\left(\begin{array}{l}
\alpha \\
\beta
\end{array}\right) \sigma(n+\beta) .
$$

from Proposition 3.1 in [7]. Using this and the facts that $a \in S_{1,0, \infty}, E_{k-1} \in$ $S_{1,0, \infty}^{-1}$ we estimate

$$
\left|\Delta_{n}^{\alpha} a(x, n)\right| \leq C 2^{|\alpha|},\left|D_{x}^{\alpha} E_{k-1}(x, n)\right| \leq C\langle n\rangle^{-1},
$$

so that each of the terms in the sum defining $T_{2}(x, n) \in S_{1,0, \infty}^{-1}$ and

$$
\left|T_{2}(x, n)\right| \leq C\langle n\rangle^{-1} .
$$

so that $T_{2}(x, n) \in S_{1,0, \infty}^{-1}$. To estimate $T_{1}(x, n)$ we define multi-indices $\alpha^{(j)}$ to be $\alpha_{r}^{(j)}=\delta_{r j}, r=1, \ldots, d$, and split $T_{1}$ as

$$
T_{1}(x, n)=\sum_{j=1}^{d} \sum_{\alpha \geq 1, \alpha_{j} \geq 1} \frac{1}{\alpha !}\left(\Delta_{n}^{\alpha-\alpha^{(j)}} \Delta_{n}^{\alpha^{(j)}} a(x, n)\right) D_{x}^{(\alpha)}(a(x, n))^{k-1} .
$$

Then clearly $\Delta_{n}^{\alpha^{(j)}} a(x, n) \in S_{1,0, \infty}^{-1}$. If $a, b \in S_{1,0}^{0}$, then $a b \in S_{1,0}^{0}$, however the same is not true for $S_{1,0, \infty}^{0}$ in view of Leibniz rule for derivatives. Therefore using the property of $a \in S_{1,0, \infty}$, we estimate

$$
\left|\left(\Delta_{n}^{\alpha-\alpha^{(j)}} \Delta_{n}^{\alpha^{(j)}} a(x, n)\right)\right| \leq C 2^{|\alpha|}\langle n\rangle^{-1}, \frac{1}{\alpha !}\left|D_{x}^{(\alpha)}(a(x, n))^{k-1}\right| \leq C^{k-1} \prod_{j=1}^{d} \theta_{j},
$$

where

$$
\theta_{j}=\left\{\begin{array}{l}
1, \quad \alpha_{j} \leq k-1, \\
\frac{1}{\left(\alpha_{j}-k+1\right) !}, \quad \alpha_{j}-k+1>0
\end{array} \quad, \quad j=1, \ldots, d,\right.
$$

Then arguing as done for the term $T_{2}(x, n)$ we see that $T_{1}(x, n) \in S_{1,0, \infty}^{-1}$ and

$$
\left|T_{1}(x, n)\right| \leq C\langle n\rangle^{-1}
$$

proving the lemma.

Acknowledgement: We thank M N Namboodiri for introducing us to the Szegö type theorems and referees for numerous comments on earlier versions of this paper that made us simplify and clarify the proofs here. 


\section{Appendix}

In this appendix we collect two theorems we use in our paper for the reader's convenience.

The first one is a Tauberian theorem of Grishin-Poenditsheva from [5].

Definition 3.1. Let $\phi$ be a positive function on the half line $[0, \infty)$. Let

$$
S=\left\{\alpha: \exists M, R \text { with } \phi(t r) \leq M t^{\alpha}, \text { for all } t \geq 1, r \geq R\right\}
$$

and

$$
G=\left\{\alpha: \exists M, R \text { with } \phi(t r) \geq M t^{\alpha}, \text { for all } t \geq 1, r \geq R\right\}
$$

Then $\alpha(\phi)=\inf S$ and $\beta(\phi)=\sup G$ are called the upper and lower Matushevskaya index of $\phi$ respectively.

Theorem 3.2. ([5], Theorem 2)

Let $m>-1$. Assume that $\varphi$ is positive measurable function on $[0, \infty)$ that does not vanish identically in any neighbourhood of infinity. Let $\Phi(r)=$

$\int_{0}^{\infty} \frac{\varphi(r t)}{(1+t)^{m+1}} d t$ be finite. Then the functions $\varphi$ and $\Phi$ have same growth at infinity if and only if $\beta(\varphi)>-1$ and $\alpha(\varphi)<m$.

Definition 3.3. A function $\varphi$ is said to be multiplicatively continuous at infinity if it satisfies

$$
\lim _{\substack{r \rightarrow \infty \\ \tau \rightarrow 1}} \frac{\varphi(\tau r)}{\varphi(r)}=1 \text { and } \lim _{\substack{\tau \rightarrow \infty \\ r \rightarrow 1}} \frac{\varphi(\tau r)}{\varphi(r)}=1 .
$$

Theorem 3.4. ([5], Theorem 8) Let $\varphi$ and $\psi$ be positive functions on $[0, \infty)$ satisfying the following conditions:

1. the functions $\varphi$ and $\psi$ do not vanish identically in any neighbourhood of infinity;

2. the function $\varphi$ is multiplicatively continuous at infinity and $\beta(\varphi)>-1$;

3. the function $\psi$ is increasing;

4. at least one of the inequalities $\alpha(\varphi)<m$ and $\alpha(\psi)<m$ holds, where $m>-1$; 
5. the functions

$$
\begin{aligned}
& \qquad \Phi(r)=\int_{0}^{\infty} \frac{\varphi(r u)}{(1+u)^{m+1}} d u \text { and } \Psi(r)=\int_{0}^{\infty} \frac{\psi(r u)}{(1+u)^{m+1}} d u \\
& \text { are finite and } \lim _{r \rightarrow \infty} \frac{\Psi(r)}{\Phi(r)}=1 \text { then } \lim _{r \rightarrow \infty} \frac{\psi(r)}{\varphi(r)}=1 .
\end{aligned}
$$

The above theorem derives asymptotic behaviour of $\varphi, \psi$ from the asymptotic behaviour of $\phi, \Psi$ by assuming additional conditions on $\varphi$ and $\psi$.

The next theorem is Theorem 1.2 of Laptev - Safarov [2].

Let $\mathcal{H}$ be a separable Hilbert space and $B$ a self adjoint operator, not necessarily bounded. Suppose that $f \in W_{\infty}^{2}(K)$ where $K=\bigcup_{0 \leq t \leq 1} t \sigma(B) \subset$ $\mathbb{R}$ and suppose $\pi_{\lambda}=E_{H}((0, \lambda))$.

With these conditions the theorem is :

(Take $A=H, P_{\lambda}=\pi_{\lambda}$ in Theorem 1.6 [2] to get the following restatement of their theorem, so as to be consistent with our notation.)

Theorem 3.5. (Theorem 1.6, [2]) Let $H>0$ and $\operatorname{rank}\left(\pi_{\lambda}\right)<\infty$. Then for all $f \in W_{\infty}^{2}(K)$ and for all $\lambda>0, r>0, \kappa \geq 0$ we have

$$
\begin{aligned}
& \mid \operatorname{Tr}\left(\pi_{\lambda} f(B) \pi_{\lambda}-\operatorname{Tr}\left(\pi_{\lambda} f\left(\pi_{\lambda} B \pi_{\lambda}\right) \pi_{\lambda}\right) \mid\right. \\
& \leq \frac{1}{2}\left\|f^{\prime \prime}\right\| N_{r}(\lambda)\left(\left\|\pi_{\lambda-r} B\right\|^{2}+\frac{\pi^{2} \lambda^{2 \kappa}}{6 r^{2}}\left\|H^{-\kappa} \pi_{\lambda-r}[H, B]\right\|^{2}\right) .
\end{aligned}
$$

\section{References}

[1] Keldys, M. V. On a Tauberian theorem, Trudy Mat. Inst. Stekloc. 38, (1951), 77-86.

[2] Laptev, A and Safarov, Yu. Szegö type limit theorems, J. Func. Anal. 138, (1996), 544-559.

[3] Laptev, A and Safarov, Yu. Error estimates in the generalized Szegö theorem, Journees Equations aux derivees partieleles, (1991), 1-7.

[4] U. Grenander and G. Szegö,Toeplitz Forms and their applications, 2nd edn. Chelsea, New Tork (1984).

[5] A. F. Grishin and I. V. Poedintseva, Towards the Keldysh Tauberian theorem, J. Math. Sci. no. 4134 (2006), 2272-2287. 
[6] D. Robert, Remarks on a paper of S. Zelditch: 'Szego limit Theorems in Quantum Mechanics", J. Funct. Anal. 53(1983), 304-308.

[7] M. Ruzhansky and V. Turunen, Quantization of Pseudo-differential operators on the torus, J. Fourier Anal. Appl., 16, 943-982 (2010).

[8] G. Szegö, On certain Hermitian forms associated with the Fourier series if positive function, comm. Sem. Math. Univ. Lund., 228-238 (1952).

[9] Barry Simon, Szegö's Theorem and Its Descendants: Spectral Theory for $L^{2}$ perturbations of Orthogonal Polynomials, Princeton University Press, Princeton (2010).

[10] D. Widder, The Laplace Transform, Princeton Univ. press (1941).

[11] S. Zelditch, Szegö limit theorems in quantum mechanics, J. Funct. Anal. 50(1983), 67-80. 